\title{
Validación de la Escala de Motivación Lectora en Estudiantes Chilenos de Enseñanza Básica
}

\section{Validation of the Motivation to Read Survey Among Chilean Elementary School Students}

\author{
Marianela Navarro, Pelusa Orellana y Paula Baldwin \\ Universidad de los Andes
}

\begin{abstract}
Son pocos los instrumentos debidamente traducidos y validados con los que se cuenta en el mundo hispano para medir aspectos motivacionales vinculados a la lectura. El estudio tuvo como objetivo validar psicométricamente la Escala de Motivación Lectora (EML) de Gambrell, Palmer, Codling \& Mazzoni (1996), compuesta de 2 subescalas: autoconcepto lector y valor de la lectura. La muestra por conveniencia estuvo compuesta por 698 escolares chilenos de $3^{\circ}$ a $5^{\circ}$ básico de 4 colegios subvencionados y particulares pagados de la Región Metropolitana de Chile; $53 \%$ fueron hombres y $47 \%$, mujeres. La traducción de la escala se realizó mediante el método de back translation. Se realizaron análisis de consistencia interna con alfa de Cronbach, de juicio de expertos y de validez de constructo, utilizando análisis factorial exploratorio y confirmatorio. Los resultados muestran que la EML es un instrumento confiable y válido en su versión en español, aportando, así, una herramienta para su uso con fínes de investigación o a nivel de aula, permitiendo a investigadores y docentes profundizar en la motivación lectora en niños de habla hispana.
\end{abstract}

Palabras clave: motivación lectora, autoconcepto lector, valoración de la lectura, análisis psicométrico

Few instruments have been suitably translated and validated for measuring motivational aspects linked to reading in the Spanish-speaking world. The present study was aimed at psychometrically validating the Gambrell, Palmer, Codling, and Mazzoni's Motivation to Read Survey (MTS; 1996), which comprises 2 subscales: reader's self-concept and value of reading. A convenience sampling process was used to recruit 698 Chilean $3^{\text {rd }}, 4^{\text {th }}$, and $5^{\text {th }}$ graders attending 4 subsidized and private schools in the Metropolitan Region of Chile; $53 \%$ of them were boys and $47 \%$ were girls. The scale was translated using the back translation method. Internal consistency (Cronbach's alpha), expert rater, and construct validity analyses were performed using exploratory and confirmatory factor analysis. Results show that the Spanish version of the MTS is a reliable and valid instrument that can be used for research and instructional purposes, allowing researchers and teachers to delve deeper into reading motivation in Spanishspeaking children.

\section{Keywords: reading motivation, self-concept, value of reading, psychometric analysis}

Ser un lector competente es un requisito esencial para insertarse en el mundo actual, donde la comprensión y procesamiento de la información son actividades básicas en cualquier campo laboral y profesional. La alfabetización funcional, es decir, la capacidad de comprender aquello que se lee y de utilizar textos escritos en diversas tareas cotidianas, es un objetivo prioritario de la educación escolar (Collins \& O'Brien, 2003; Organisation for Economic Co-operation and Development [OECD], 2000) y de ello se desprende el interés por investigar acerca de la lectura, su desarrollo e impacto en la sociedad. Este interés también se ve reflejado en estudios de gran escala, como son las mediciones del Programa Internacional para la Evaluación de los Estudiantes (PISA), de la Encuesta Internacional sobre Alfabetización de Adultos (IALS), del Estudio Internacional de Progreso en Comprensión Lectora (PIRLS) y del Tercer Estudio Regional Comparativo y Explicativo (TERCE), y evidentemente en la preocupación de los gobiernos locales por mejorar los niveles de desempeño lector de sus estudiantes.

En los últimos años ha habido un mayor foco de estudio en los aspectos afectivos asociados a la competencia lectora, lo que también se ha reflejado en el interés por recopilar información acerca de su estado a través de pruebas internacionales. La evidencia a partir de las pruebas PISA ha indicado, por ejemplo, que en los países de la OECD aproximadamente dos tercios de los estudiantes evaluados dicen leer por gusto

Marianela Navarro y Pelusa Orellana, Facultad de Educación, Universidad de Los Andes, Santiago, Chile; Paula Baldwin, Instituto de Literatura, Universidad de los Andes, Santiago, Chile.

Las autoras agradecen a las investigadoras Linda Gambrell, Barbara Martin Palmer, Rose Marie Codling y Susan Anders Mazzoni por autorizar el uso de la Escala de Motivación Lectora en el presente estudio y su publicación en su versión en español.

La correspondencia relativa a este artículo debe ser dirigida a Marianela Navarro, Facultad de Educación, Universidad de Los Andes, Monseñor Álvaro del Portillo 12.455, Las Condes, Santiago, Chile. E-mail: mnavarroc@uandes.cl 
diariamente (OECD, 2011). Sin embargo, también se muestra que este porcentaje ha ido disminuyendo desde la década pasada y que las niñas y los estudiantes provenientes de entornos socioeconómicamente más privilegiados destinan más tiempo a la lectura recreativa que sus pares varones y de entornos más vulnerables. Esta brecha socioeconómica y de género además parece ir aumentando. Finalmente, el informe de la OECD afirma que la lectura recreativa, que es la que realizan los lectores motivados en forma permanente, está asociada con un mejor desempeño en comprensión lectora en las pruebas PISA (OECD, 2011).

La realidad chilena no difiere significativamente de lo observado por la OECD en otros países. El desempeño de estudiantes chilenos en PISA, si bien ha mejorado en forma importante a partir de 2000, aún está lejos del logro promedio de países de similar desarrollo económico. Y aun cuando estos resultados se refieren a adolescentes, la prueba de lectura del Sistema de Medición de la Calidad de la Educación (SIMCE) también muestra un bajo nivel de alfabetización funcional en los estudiantes de educación básica. En estos resultados también se aprecian diferencias importantes en cuanto a género y nivel socioeconómico (NSE), y lo mismo ocurre respecto de la motivación a la lectura. Más específicamente, al analizar la evolución de los resultados SIMCE desde el año 2003 al 2015, el puntaje de los varones cayó 12 puntos, mientras que el de las mujeres permaneció estable. En el caso de los hombres, el retroceso más importante se produjo en el NSE alto y medio alto (-37 y -32 puntos, respectivamente). Una posible explicación se relaciona con aspectos motivacionales y con hábitos de lectura, pues un cuestionario que se aplica en conjunto con el SIMCE mostró que el $50 \%$ de los hombres no lee por entretenimiento, un $46 \%$ lee solo si es obligado y un $25 \%$ aprecia recibir un libro de regalo, versus 30, 29 y 50\% en el caso de las mujeres, respectivamente (Agencia de la Calidad de la Educación, 2016a).

Otros estudios de gran escala dan cuenta del desinterés lector de la población chilena. Uno de ellos es la encuesta Chile y los Libros que realiza desde 2006 la Fundación La Fuente y Adimark GFK a mayores de 18 años; en sus tres aplicaciones se ha observado que más de la mitad de los chilenos afirma no leer libros (Fundación La Fuente/Adimark GFK, 2010). En ella también aparecen brechas de género y NSE respecto del tiempo destinado a la lectura, el acceso a libros y la valoración general del hábito lector. Un segundo estudio realizó en 2014 la Dirección de Estudios Sociales de la Universidad Católica e incluyó a una población entre 9 y 14 años y otra de 15 a 65 en más de 300 comunas de Chile; su objetivo era indagar acerca del comportamiento lector de los chilenos. En el segmento 9 a 17 años, solo un 44\% declara leer diariamente durante 15 a 20 minutos. Un $47 \%$ de ellos dice no leer por gusto (Consejo Nacional de la Cultura y las Artes, 2014). El mismo organismo realizó un estudio previo que mostró que dentro de los menores de 15 años solo un 5\% prefiere leer en su tiempo libre. Respecto del valor que las personas asignan a la lectura, los resultados muestran que solo el 14\% de los encuestados la valora como una actividad de entretención o tiempo libre (Consejo Nacional de la Cultura y las Artes \& Centro de Microdatos de la Universidad de Chile, 2011). Finalmente, la OECD realizó un estudio comparativo entre 34 países, denominado Evaluación Internacional de las Competencias de Adultos (PIAAC) en personas de 16 a 65 años de edad. Los resultados muestran que $53 \%$ de los chilenos evaluados presenta un bajo nivel de comprensión lectora, versus un 19\% promedio de los países OECD. Según el informe, esto se traduce en menor empleabilidad y productividad para el país (OECD, 2016).

En cuanto a los esfuerzos por desarrollar o validar instrumentos para evaluar la motivación lectora en Chile, Muñoz Valenzuela et al. (2012) dan cuenta de la validación de la Escala de Motivación por la Lectura Académica (EMLA), basada en la teoría de expectativa-valor de Eccles y Wigfield (2002), pero que no incluye la lectura recreativa, y que fue aplicado y validado en una muestra de estudiantes universitarios. No se han realizado en Chile, al menos en lo que respecta a nuestro conocimiento, validaciones de instrumentos que examinen la lectura recreativa ni tampoco que tomen en consideración alumnos en edad escolar.

Ante esta panorámica, es evidente que en Chile hay un problema de lectura que no solo se vincula a las habilidades lectoras, sino también al interés o motivación por leer en forma recreativa. La principal preocupación deriva precisamente de la alta correlación entre las habilidades y la motivación lectora, que en el caso chileno pareciera no darse de manera tan significativa. Por lo mismo, resulta interesante poder contar con una mayor cantidad de información acerca de la valoración que los niños y jóvenes chilenos le asignan a la lectura como actividad de tiempo libre, así como también acerca de sus propias prácticas lectoras. Un desafío importante para lograr este objetivo es contar con instrumentos válidos y confiables que puedan aplicarse a hablantes del idioma español que aborden los aspectos afectivos vinculados al hábito lector de manera más acotada. 


\section{Aproximaciones Teóricas a la Motivación Lectora}

La relación entre el desempeño lector y la motivación por esta actividad ha relevado la importancia de la motivación lectora como aspecto fundamental para incrementar los niveles de competencia en la lectura (Santa et al., 2000). Una consecuencia importante del fracaso lector es el bajo nivel de interés por leer (Hiebert, 2009; Morgan, Fuchs, Compton, Cordray \& Fuchs, 2008). Los lectores motivados, por el contrario, además de ser competentes, leen con mayor frecuencia y suelen mencionar la lectura como pasatiempo valorado (Guthrie, Wigfield, Metsala \& Cox, 1999; Wigfield \& Guthrie, 1997). Algunos estudios han demostrado que la motivación lectora contribuye de manera importante al buen desempeño en comprensión lectora, especialmente en el caso de los alumnos de $3^{\circ}$ a $5^{\circ}$ año básico, confirmándose incluso su poder predictivo en pruebas estandarizadas de lectura (Gottfried, 1985).

Dada su multidimensionalidad, los enfoques teóricos acerca de la motivación lectora son numerosos (Malloy \& Gambrell, 2008). Esencialmente, las teorías acerca de la motivación lectora se centran en explicar los factores que determinan la elección de la lectura como actividad de tiempo libre, la valoración que las personas hacen de esta actividad por sobre otras o la manera en que los lectores se perciben a ellos mismos. Entre las teorías motivacionales de la lectura, aquellas que toman en cuenta aspectos tales como el autoconcepto lector y la valoración de la lectura son frecuentemente citadas en la literatura. Una de ellas es la teoría de la expectativa-valor (EVT, por su nombre en inglés expectancy-value theory), desarrollada por Eccles y Wigfield (2002). Estos autores plantean que la motivación hacia la lectura se ve influenciada por dos aspectos centrales: la manera en que un lector se percibe a sí mismo y el grado de apreciación que expresa frente a la actividad de leer. Ambos constructos aparecen en otros enfoques teóricos, por ejemplo, McKenna, Kear y Ellsworth (1995) asignan un valor importante a los aspectos afectivos y a las creencias en torno a la lectura, los que aparecen asociados a una actitud positiva hacia la misma. EI contexto social impacta y da forma a las creencias, opiniones y expectativas acerca de la lectura (Unrau \& Schlackman, 2006), lo que se manifiesta en expectativas, propósitos de lectura y la intención de leer.

Por otra parte, existen modelos teóricos que distinguen entre motivación intrínseca y extrínseca (Deci, 1992) y que suelen utilizarse para analizar los intereses lectores de los escolares. Desde esta perspectiva, los lectores que se motivan intrínsecamente son aquellos que eligen leer por decisión propia y no impuesta y que perciben la lectura como placentera y valiosa (Becker, McElvany \& Kortenbruck, 2010; Guthrie \& Klauda, 2014; Pintrich \& Schunk, 2002). Los lectores intrínsecamente motivados suelen mostrar curiosidad e involucramiento frente a la lectura y esta forma parte de sus actividades de tiempo libre. Por otra parte, la motivación extrínseca se refiere a la decisión de leer ante la oportunidad de recibir algún tipo de recompensa externa por hacerlo. Quienes están extrínsecamente motivados por leer lo hacen, porque saben que serán reconocidos por hacerlo, ya sea mediante una nota, premio o valoración social, y no necesariamente por considerar la lectura como una actividad placentera o valorada (Schiefele, Schaffner, Moller \& Wigfield, 2012). La evidencia muestra que quienes exhiben una motivación extrínseca por la lectura tienden a desarrollar estrategias de comprensión más superficiales y su motivación no trasciende más allá de la obtención de una recompensa externa.

Junto con mencionar los enfoques teóricos desde los cuales se ha analizado el tema de la motivación lectora, no podemos dejar de agregar aspectos contextuales y culturales que definitivamente inciden en favorecer su desarrollo. En el caso de la realidad chilena, aspectos tales como el acceso a libros y la consideración de los intereses y gustos de los lectores en la selección de material literario son dos ámbitos de importancia. Varias investigaciones han confirmado que para los niños de escasos recursos la falta de libros incide directamente en una menor proporción de horas dedicadas a leer (Neuman \& Celano, 2001; Van Slyck, 1995). Las encuestas sobre motivación lectora realizadas en Chile han identificado la falta de libros y su alto costo como razones por las cuales los chilenos no leen en su tiempo libre. Según el Estudio sobre el Comportamiento Lector (Consejo Nacional de la Cultura y las Artes \& Centro de Microdatos de la Universidad de Chile, 2011), si bien más del 90\% de los encuestados dice tener libros en su casa, solo el 13\% tiene más de 100 libros. Por otra parte, el estudio de la Fundación La Fuente/Adimark GFK (2010) mostró que el $80 \%$ de los chilenos cree que los libros son muy caros, y el NSE de los encuestados fue el principal diferenciador de la cantidad de libros en el hogar. Respecto de la posibilidad de incluir los intereses y gustos de los lectores dentro de la selección de material, ha habido esfuerzos importantes en este sentido, en particular en las bibliotecas de aula que el Ministerio de Educación entrega a los establecimientos educacionales mediante los Centros de Recursos para el Aprendizaje. Estas bibliotecas han aumentado la 
cantidad de títulos por establecimiento y por alumno, lo que ha contribuido a entregar una oferta más variada de títulos en cada colección (Chile, Ministerio de Educación, 2013). Sin embargo, se hace necesario contar con instancias que permitan captar los intereses y gustos literarios de los usuarios de estas bibliotecas, considerando no solo la diversidad temática sino también el género, formato, extensión y nivel de complejidad.

Chile ha realizado esfuerzos importantes para fomentar la lectura a través de planes, capacitaciones docentes, talleres e iniciativas públicas y privadas orientadas a potenciar el gusto por la lectura. La más reciente ha sido el Plan Nacional de Fomento a la Lectura: Lee Chile Lee, que comenzó con planes de animación a la lectura pensados para niños pequeños (Arnold \& García Céspedes, 2012). Junto con capacitar a las educadoras en la lectura de cuentos infantiles, se entregaron sets de libros y material de apoyo a la animación lectora. La evaluación de estos programas muestra que la mitad de las educadoras de párvulos del 83\% de los jardines en los que se implementan bibliotecas lee con los niños todos los días (Arnold \& García Céspedes, 2012). Programas como estos también se desarrollan en los niveles superiores de educación, abordando principalmente los objetivos de capacitar a los docentes en la lectura de libros y dotar a las bibliotecas y aulas de colecciones más atractivas para los alumnos.

\section{La Relación entre la Motivación a la Lectura y el Rendimiento Lector}

Numerosas investigaciones han confirmado que existe una relación entre la motivación por leer y el desempeño lector (Morgan \& Fuchs, 2007; Wang \& Guthrie, 2004; Wigfield \& Guthrie, 1997). Esta relación parece estar también mediada por la frecuencia lectora (Guthrie et al., 1999), indicando que los lectores motivados leen con mayor frecuencia y que la lectura frecuente facilita el desarrollo de competencias lectoras más avanzadas. Estos lectores competentes suelen tener un alto sentido de autoeficacia que, a su vez, les hace valorar las tareas de lectura, cosa que no ocurre entre aquellos lectores que, al tener menores habilidades para leer, podrían desmotivarse rápidamente y con ello dejar de leer, lo que generaría un círculo vicioso que les impediría tener oportunidades de mejorar dichas habilidades. Ello explicaría que en muchos casos se ha identificado la desmotivación por la lectura como una de las causas subyacentes al bajo nivel de desempeño lector, así también como la bidireccionalidad en la relación existente entre estos dos constructos (Morgan \& Fuchs, 2007).

Estudios más recientes se han focalizado en analizar el rol predictor de la motivación en la competencia lectora, intentando determinar el porcentaje de varianza en comprensión que podría explicarse a partir del interés y gusto lector. En la educación básica, por ejemplo, se ha encontrado gran variabilidad en el porcentaje de varianza explicada por la motivación, lo que tendría su justificación tanto en los enfoques teóricos de la motivación como también en los instrumentos usados para medirla (Cartwright, Marshall \& Wray, 2016; Taboada, Tonks, Wigfield \& Guthrie, 2009). No encontramos, sin embargo, estudios que analicen el rol de la motivación en el aumento de la comprensión lectora en estudiantes de habla hispana ni, más específicamente, en la población chilena. Lo anterior no hace sino constatar la necesidad de abordar la relación motivacióndesempeño lector, sobre todo considerando que los estudios y mediciones internacionales destacan el factor motivacional como un componente esencial de la alfabetización, incluso más influyente que el NSE (Kirsch et al., 2002).

\section{La Escala de Motivación Lectora}

La Escala Motivation to Read fue desarrollada por Linda Gambrell, Barbara Palmer, Rose Marie Codling y Susan Mazzoni en 1996 y es uno de los instrumentos más usados para evaluar la motivación lectora. Se fundamenta en las teorías motivacionales de Eccles (1983), quien establece que la motivación se ve fuertemente influenciada por las expectativas de éxito que la persona tiene frente a una determinada tarea, así como también por la valoración que el sujeto hace de dicha tarea. Desde la perspectiva de la lectura, esta mirada teórica está sustentada en estudios que muestran que cuando los lectores se perciben como competentes tienden a obtener mejores niveles de desempeño que cuando no tienen confianza en sus habilidades (Chapman \& Tunmer, 1995; Schunk, 1985). De la misma forma, respecto de la valoración de la lectura, hay estudios que han comprobado que los lectores que valoran el acto de leer tienden a dedicarle más tiempo y, por ende, a desarrollar mejores habilidades de comprensión (Guthrie et al., 2004). Como se desprende de lo anterior, se trata de un enfoque de motivación intrínseca (Schunk, Pintrich \& Meece, 2008), en el que las disposiciones hacia la lectura se fundamentan en razones o valoraciones de la persona y no de su entorno (Schaffner, Philipp \& Schiefele, 2016; Wigfield \& Guthrie, 1997). 
Por otra parte, el creciente interés por los factores afectivos vinculados al aprendizaje ha generado especial interés en el ámbito del desempeño lector (Afflerbach \& Cho, 2011; Hidi \& Harackiewicz, 2000). De hecho, estudios acerca de la motivación y su relación con el buen desempeño escolar han logrado explicar hasta un $11 \%$ de la varianza del rendimiento por factores motivacionales (Uguroglu \& Walberg, 1979) y más recientemente se ha establecido que la correlación entre la habilidad verbal y el autoconcepto de dicha habilidad es $r=0,49$ (Möller, Pohlmann, Köller \& Marsh, 2009). Este tipo de resultados explica el surgimiento de teorías acerca de la motivación que se fundamentan en la relación entre expectativas y valoración, la autodeterminación, entre otras (Pintrich \& Schunk, 2002).

La Escala de Motivación Lectora (EML) forma parte del Perfil de Motivación Lectora, que incluye, además, una entrevista semiestructurada. Es un cuestionario de auto reporte que puede ser aplicado en forma grupal o individual. En su versión original se compone de 20 ítems que contienen aseveraciones acerca del comportamiento lector y que deben responderse en función de una escala de cuatro puntos. El instrumento se diseñó pensando en alumnos de $2^{\circ}$ año básico en adelante. Los 20 ítems seleccionados surgieron de un banco de más de 100 ítems evaluados en función de la validez de constructo y juicio de expertos (Gambrell et al., 1996). La versión final del instrumento fue validada en una aplicación piloto a 330 estudiantes de $3^{\circ}$ y $5^{\circ}$ año básico en los Estados Unidos mediante análisis factorial exploratorio (AFE), usando el método de mínimos cuadrados ponderados y rotación ortogonal varimax. Para la consistencia interna se utilizó alfa de Cronbach, la cual se estimó en 0,75 para auto concepto lector y 0,82 para valoración de la lectura. Los índices de confiabilidad pre y post test también resultaron robustos, lo que ratificó la confiabilidad del cuestionario (Gambrell et al., 1996).

Como ya mencionamos, la escala es probablemente una de las más utilizadas en estudios acerca de motivación lectora infantil. Una revisión realizada por los autores en 2013 permitió modificar algunos ítems y reconfigurar el texto de las alternativas en la escala Likert (Malloy, Marinak, Gambrell \& Mazzoni, 2013). También se realizaron nuevos análisis psicométricos, aunque no se incursionó en la validez estructural del instrumento. Más tarde, Watkins y Browning (2015) publicaron un análísis que incluyó la validez estructural y que también realizó cambios en algunos de los ítems que no mostraron relación con los constructos previamente definidos. Posteriormente y mediante análisis factorial confirmatorio (AFC) lograron demostrar el calce con un modelo de dos factores (Watkins \& Browning, 2015). No encontramos evidencia de la validación de la escala de Gambrell et al. en Chile ni en otros países de habla hispana.

Existen pocos estudios que validen esta escala a nivel internacional, pese a que es ampliamente utilizada en estudios de motivación. En la presentación de la escala las autoras (Gambrell et al., 1996) no entregan mayores detalles del AFE realizado; solo dan a conocer el método de extracción de factores y de rotación. Por otra parte, este análisis ha sido, además, cuestionado por Watkins y Browning (2015), quienes sostienen que la rotación ortogonal en el AFE utilizada por Gambrell et al. (1996) no considera la posible correlación entre las subescalas de autoconcepto lector y valor de la lectura. Si bien es cierto que las autoras (ver, por ejemplo, Malloy et al., 2013) y otros (Watkins \& Browning, 2015) realizaron nuevos estudios de validez, estos incluyeron solo un AFC. Es por esta razón que creemos que la información que entregue un estudio de validación que incluya AFE y AFC de su versión en español puede contribuir a establecer de manera más sólida la yalidez de la EML, especialmente en el contexto hispanoparlante.

Otros instrumentos para medir el grado de motivación lectora son el Motivation to Read Questionnaire (MRQ) desarrollado por Wigfield y Guthrie (1997) y la Elementary Reading Attitude Survey, desarrollada por Michael McKenna y Dennis Kear (1990). El primero aborda varios ámbitos de la lectura, como son la eficacia, el desafío de la lectura, la curiosidad, el nivel de involucramiento y la importancia, entre otros. Sin embargo, como incluye 53 ítems, resulta considerablemente más extensa de aplicar y se utiliza con alumnos entre $4^{\circ} \mathrm{y}$ $8^{\circ}$ año básico. El segundo instrumento es similar al de Gambrell et al. (1996) en cuanto a que tiene 20 ítems y que también utiliza escala Likert para las respuestas, aunque estas se presentan en forma icónica, es decir, hay cuatro opciones de estados de ánimo graficados en la figura del conocido gato Garfield, y el alumno debe escoger la que mejor representa sus sentimientos respecto de la pregunta. La evidencia respecto de la estabilidad del instrumento y su validez ha sido variada, aunque se observan buenos niveles de consistencia interna (e.g., Kazelskis et al., 2005). Ambos instrumentos están desarrollados en inglés y no se dispone de traducciones validadas en idioma español.

Las razones que justifican la validación de la EML de Gambrell et al. son varias. En primer lugar, es un instrumento que resulta atractivo, considerando su facilidad de acceso y simpleza en la aplicación, tabulación 
y análisis. En segundo lugar, las propiedades psicométricas de la escala aplicada en países de habla inglesa, así como en otros lugares como Finlandia y Grecia (Gambrell et al., 2013, Agosto), dan cuenta de la estabilidad de los puntajes que el instrumento entrega, su confiabilidad y validez de constructo. Finalmente, y desde un punto de vista teórico, el abordaje de la motivación lectora desde los constructos de autoconcepto lector y valoración de la lectura puede entregar información interesante respecto del fenómeno de la motivación lectora entre nuestros estudiantes. Comprender mejor el grado de asociación entre la forma en que los lectores se perciben respecto del hábito de leer y el valor que le asignan a este acto puede ayudarnos a establecer políticas de incentivo a la lectura y a generar intervenciones pedagógicas tendientes a fortalecer tanto la percepción de las habilidades como la importancia de la lectura en la vida de las personas.

\section{Método}

\section{Participantes}

Los participantes fueron 698 estudiantes de $3^{\circ}, 4^{\circ}$ y $5^{\circ}$ año básico pertenecientes a cuatro establecimientos educacionales de la Región Metropolitana de Chile. La muestra corresponde a una selección no probabilística por conveniencia, aunque se resguardó la participación de establecimientos de al menos dos dependencias administrativas (subvencionada y particular pagada), de un número similar de estudiantes de sexo femenino y masculino y de números similares de participantes de cada curso. Del total de estudiantes, $34 \%$ son de $3^{\circ}$ $(n=242)$, $34 \%$ son de $4^{\circ}(n=235)$ y $32 \%$ son de $5^{\circ}(n=221)$. En cuanto a la distribución por sexo, $53 \%$ son hombres $(n=367)$ y $47 \%$ son mujeres $(n=331)$. Según el tipo de dependencia administrativa, $52 \%$ de los estudiantes pertenecen a establecimientos de tipo particular pagado $(n=362)$ y $48 \%$ a escuelas particulares subvencionadas $(n=336)$.

\section{Instrumento}

La EML consta de 20 ítems (ver Tabla 1), 10 de los cuales se utilizan para evaluar el autoconcepto lector (todos los ítems impares) y 10, para la valoración de la lectura (todos los ítems pares). Se trata de ítems de opción múltiple, donde los enunciados son abiertos, que se completan con las cuatro opciones de respuesta, las cuales combinan respuestas graduadas de diferentes tipos: frecuencia (de Siempre a Nunca), calidad (de Muy bien a Muy mal), cantidad (de Casi todo a Casi nada) e importancia (de Muy importante a No muy importante). La mitad de los ítems presenta las opciones ordenadas de la opción más positiva a la más negativa y la otra mitad en forma inversa para evitar respuestas estereotipadas.

Tradujimos la EML del inglés al español usando el método de back translation (Brislin, 1970), en el que primeramente un traductor bilingüe realiza una traducción del inglés original al español y luego se le pide a otro traductor bilingüe que vuelva a traducir dicha versión al inglés. Posteriormente se comparan ambas versiones en inglés para corroborar la acuciosidad en la terminología. Una tercera revisión estuvo a cargo de un panel de expertos, quienes compararon la versión original en inglés con la traducción al español. También revisaron el documento para corroborar que el texto en español fuera comprensible para niños de educación básica y que no existieran términos culturalmente foráneos que dificultasen la respuesta. La versión final de la escala fue aplicada en papel.

Una vez traducido el instrumento, convocamos a seis educadores para realizar el juicio de expertos como parte del proceso de validación. Para ello, pedimos a los jueces revisar cada uno de los ítems de la escala y determinar a cuál de los dos constructos teóricos (autoconcepto lector o valoración de la lectura) se ajustaban mejor. Adicionalmente, les pedimos que evaluaran el grado de importancia que le asignaban a cada ítem dentro de la escala.

La versión traducida al español se encuentra en el Anexo, incluidas las instrucciones sobre la puntuación.

\section{Procedimiento}

Encuestamos a los estudiantes en mayo de 2014. Previo a ello, contactamos a los colegios y sostuvimos reuniones con los directivos de cada uno de ellos para explicarles los objetivos y procedimientos del estudio. Obtuvimos las autorizaciones correspondientes de parte de la dirección de los establecimientos, así como también los consentimientos informados de parte de los apoderados. La aplicación del instrumento se realizó por profesores con estudios de postgrado, quienes fueron capacitados en el uso y procedimiento de 
administración. La aplicación se realizó en formato de lápiz y papel y en forma grupal en los distintos cursos; los estudiantes demoraron alrededor de 20 minutos en completarlo.

La participación de los estudiantes fue voluntaria y respaldada mediante la firma de consentimientos informados de parte de los padres, los que enviamos a cada establecimiento. Los estudiantes rellenaron la escala con nombre y apellido, pues los colegios solicitaron tener esa información en los informes finales que elaboramos para cada uno de ellos. Sin embargo, para fines de publicación y para proteger la confidencialidad de los datos, tanto individuales como agregados, se tabularon sin utilizar nombres, sino números y códigos.

Asimismo, los aspectos éticos de esta investigación han sido analizados y revisados por el comité de ética de la institución de afiliación de las autoras.

\section{Análisis de Datos}

Calculamos la consistencia interna mediante alfa de Cronbach, para la escala completa y cada subescala (autoconcepto lector y valor de la lectura), y evaluamos el aporte de cada ítem a la misma. Verificamos la validez de constructo utilizando dos métodos: AFE y AFC. Para este propósito, dividimos la muestra en dos partes al azar: 345 y 353 casos para el AFE y el AFC, respectivamente. Para el AFE analizamos la bondad de ajuste de un modelo factorial a los datos mediante la medida de adecuación muestral Kaiser-Meyer-Olkin (KMO) y la prueba de esfericidad de Barlett. Asimismo, para el AFE utilizamos ejes principales como método de extracción de los factores y rotación oblicua promax. Decidimos utilizar rotación no ortogonal bajo el supuesto de que los factores se encuentran correlacionados. Para la selección del número de factores consideramos el gráfico de sedimentación, la regla de Kaiser, que indica conservar los factores cuyos valores propios son $\geq 1$, el porcentaje de varianza explicada por cada factor y la interpretación sustantiva de los factores obtenidos. Realizamos el AFE mediante SPSS versión 21. El AFC tiene el propósito de evaluar el ajuste del modelo teórico propuesto por Gambrell et al. (1996) a los datos. Asimismo, comparamos el modelo teórico con tres modelos alternativos. La evaluación de los modelos la realizamos mediante el estadístico $\chi^{2}$ y la razón de este valor con sus grados de libertad. Sin embargo, considerando la sensibilidad de esta prueba al tamaño muestral (Cumsille, Martínez, Rodríguez \& Darling, 2014; Widaman, Ferrer \& Conger, 2010), analizamos, además, el índice de bondad de ajuste de Joreskog (GFI), la raíz del error cuadrático medio de aproximación (RMSEA) y la raíz del residuo cuadrático medio estandarizado (SRMR). Para evaluar un ajuste adecuado consideramos los siguientes parámetros: valor entre 0 y 3 para la razón entre $\chi^{2}$ y sus grados de libertad, valor superior a 0,90 para GFI y valor inferior a 0,08 para RMSEA y SRMR (Bentler, 1990). Realizamos el AFC con el Paquete $\mathrm{R}$ lavaan para modelo de ecuaciones estructurales, utilizando el método de máxima verosimilitud (Rosseel, 2012).

\section{Análisis de Juicio de Expertos}

\section{Resultados}

El análisis de las evaluaciones de los expertos muestra que hay entre un $100 \%$ y un $84 \%$ de concordancia entre los jueces para ocho de los ítems asignados al constructo de autoconcepto lector (ítems 1, 3, 7, 9, 13, 15, 17 y 19) y para siete de los ítems asociados a valoración de la lectura (ítems 4, 8, 10, 12, 14, 18, y 20). El ítem que obtiene un menor porcentaje de concordancia es el ítem 11, ya que un $57 \%$ de los jueces lo adscribe al constructo de autoconcepto y el $43 \%$ restante, al de valoración.

\section{Análisis de Consistencia Interna}

La EML mostró una buena consistencia interna a nivel global $(\alpha=0,82)$, así como a nivel de las subescalas de autoconcepto lector $(\alpha=0,71)$ y valor de la lectura $(\alpha=0,77)$. En cuanto al aporte que realiza cada ítem a la consistencia interna total, solo el ítem 11 resultó ser un reactivo que, de eliminarse, aumentaría ligeramente la consistencia de la escala global $(\alpha=0,83)$ y de la subescala de autoconcepto, que es a la que teóricamente pertenece $(\alpha=0,74)$. 


\section{Análisis Factorial Exploratorio}

Las medidas de bondad de ajuste, $\mathrm{KMO}=0,85$ y prueba de esfericidad de Barlett, $\chi^{2}(190, N=345)=1446,87$, $p<0,001$, mostraron una buena adecuación de un modelo factorial a los datos.

La solución que escogimos, según los criterios que definimos en el Método, presenta dos factores, los que dan cuenta de un $26 \%$ de la varianza explicada del autoconcepto lector y la valoración de la lectura.

El AFE muestra dos factores (ver Figura 1), los que se corresponden con las subescalas de la EML: Factor 1 (autoconcepto lector) y Factor 2 (valor de la lectura).

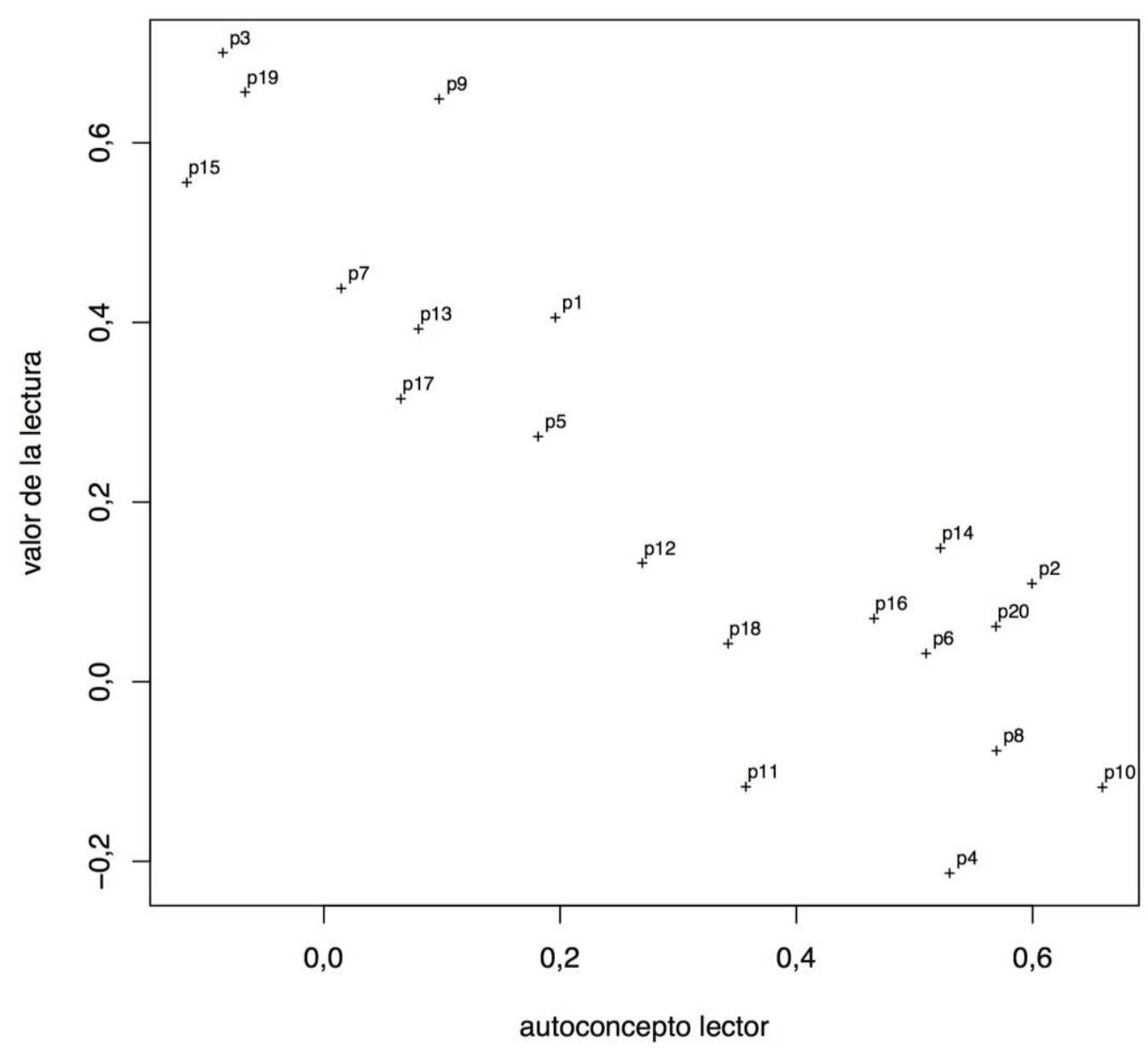

Figura 1. Pesos por factor. En el eje X se indican los pesos correspondientes al Factor 1 (autoconcepto lector) y en el eje Y los pesos correspondientes al Factor 2 (valor de la lectura)

Presentamos las cargas factoriales del AFE en la Tabla 1. Para facilitar su interpretación, suprimimos las cargas factoriales $<0,25$. Los ítems cargan en su mayoría en su subescala teórica, a excepción del ítem 11 que carga en la subescala opuesta. Luego, el ítem 12 presenta un peso factorial inferior a 0,25.

\section{Análisis Factorial Confirmatorio}

La Tabla 1 muestra también el resultado del AFC, según el modelo teórico de Gambrell et al. (1996), el cual muestra bastante coincidencia con el AFE en cuanto a los ítems que resultaron problemáticos. El ítem 11 no presenta una carga factorial significativa; sin embargo, el ítem 12 alcanza una carga factorial 0,30 en el factor teórico al cual corresponde. La información de la Tabla 1 se complementa con la de la Figura 2, en la 
cual se observa el modelo teórico, las cargas factoriales y el error asociado a la estimación de los pesos factoriales.

Tabla 1

AFE y AFC de la EML de Gambrell et al. (1996)

\begin{tabular}{|c|c|c|c|c|c|}
\hline \multirow{2}{*}{$\begin{array}{l}\mathrm{N}^{\circ} \\
\text { item }\end{array}$} & \multirow[b]{2}{*}{ Ítem } & \multicolumn{2}{|c|}{$\mathrm{AFE}$} & \multicolumn{2}{|c|}{$\mathrm{AFC}$} \\
\hline & & $\begin{array}{c}\text { Autoconcepto } \\
\text { lector }\end{array}$ & $\begin{array}{l}\text { Valor de la } \\
\text { lectura }\end{array}$ & $\begin{array}{c}\text { Autoconcepto } \\
\text { lector }\end{array}$ & $\begin{array}{c}\text { Valor de la } \\
\text { lectura }\end{array}$ \\
\hline 1 & Mis amigos creen que soy... (-) & 0,52 & & 0,49 & \\
\hline 3 & Yo leo... & 0,70 & & 0,61 & \\
\hline 5 & $\begin{array}{l}\text { Cuando me encuentro con una palabra } \\
\text { que no conozco puedo... (-) }\end{array}$ & 0,37 & & 0,32 & \\
\hline 7 & Cuando leo solo comprendo... (-) & 0,39 & & 0,27 & \\
\hline 9 & Yo soy... & 0,65 & & 0,63 & \\
\hline 11 & $\begin{array}{l}\text { Me preocupa lo que los otros niños } \\
\text { piensen de mi lectura... (-) }\end{array}$ & -- & 0,30 & -. & \\
\hline 13 & $\begin{array}{l}\text { Cuando el profesor me pregunta sobre } \\
\text { lo que yo he leído... }\end{array}$ & 0,52 & & 0,42 & \\
\hline 15 & Leer es... (-) & 0,54 & & 0,34 & \\
\hline 17 & $\begin{array}{l}\text { Cuando estoy en un grupo contando } \\
\text { historias... }\end{array}$ & 0,33 & & 0,38 & \\
\hline 19 & Cuando yo leo en voz alta leo... & 0,59 & & 0,50 & \\
\hline 2 & $\begin{array}{l}\text { Leer un libro es algo que a mí me } \\
\text { gusta hacer... }\end{array}$ & & 0,52 & & 0,55 \\
\hline 4 & $\begin{array}{l}\text { Mis mejores amigos piensan que leer } \\
\text { es... (-) }\end{array}$ & & 0,47 & & 0,38 \\
\hline 6 & $\begin{array}{l}\text { Yo le cuento a mis amigos sobre los } \\
\text { buenos libros para leer... }\end{array}$ & & 0,48 & & 0,52 \\
\hline 8 & Las personas que leen mucho son... (-) & & 0,50 & & 0,46 \\
\hline 10 & Yo creo que las bibliotecas son... (-) & & 0,71 & & 0,57 \\
\hline 12 & Saber leer bien es... & & -- & & 0,30 \\
\hline 14 & Creo que leer es... & & 0,57 & & 0,64 \\
\hline 16 & Cuando sea grande yo... & & 0,49 & & 0,48 \\
\hline 18 & $\begin{array}{l}\text { Me gustaría que mi profesor nos leyera } \\
\text { libros en voz alta en clase... (-) }\end{array}$ & & 0,38 & & 0,37 \\
\hline 20 & $\begin{array}{l}\text { Cuando me regalan un libro me } \\
\text { siento... (-) }\end{array}$ & & 0,50 & & 0,50 \\
\hline
\end{tabular}

Nota. (-) indica que el ítem fue codificado de forma inversa; AFE: análisis factorial exploratorio; AFC: análisis factorial confirmatorio; --: peso factorial $<0,25$.

Probamos tres modelos alternativos al modelo teórico (ver Tabla 2), en los que eliminamos progresivamente los dos ítems que resultaron problemáticos, tanto en el AFE como en el AFC. Todos los modelos presentan una razón entre $\chi^{2}$ y sus grados de libertad $\leq 3$ y esta magnitud decrece en la medida en que vamos eliminando del modelo aquellos ítems problemáticos. La bondad de ajuste, representada por el índice GFI, es menor al parámetro esperado $(>0,90)$, cuando se trata del modelo teórico, y mayor en los modelos alternativos testeados, aunque aumenta ligeramente en el Modelo 2. Asimismo, los índices RMSEA y SRMR son inferiores a 0,08. En síntesis, de los modelos testeados, el Modelo 2 ( $\sin l o s$ ítems 11 y 12) resulta ser el de mejor ajuste a los datos. 


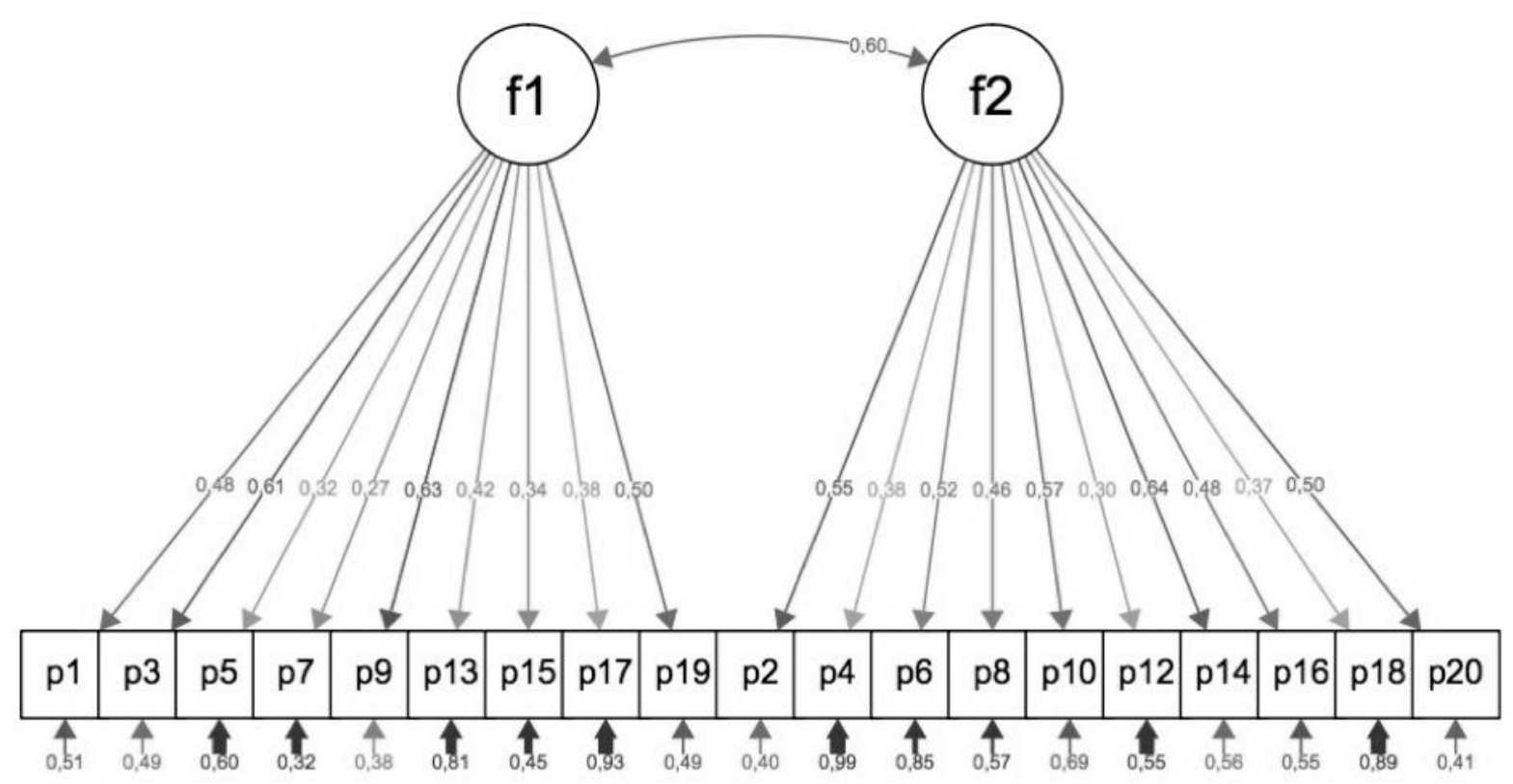

Figura 2. Modelo teórico de la EML de Gambrell et al. (1996). f1: Factor 1 (autoconcepto lector), f2: Factor 2 (valor de la lectura)

Índices de Bondad de Ajuste del AFC

\begin{tabular}{lccccccc}
\hline Modelo & $\chi^{2}$ & $g l$ & $\chi^{2} / g l$ & $p$ de $\chi^{2}$ & GFI & RMSEA & SRMR \\
\hline Modelo teórico & 3240,31 & 169 & 10,919 & $<0,001$ & 0,884 & 0,051 & 0,059 \\
Modelo 1 (sin 11) & 2650,53 & 151 & 10,758 & $<0,001$ & 0,912 & 0,046 & 0,053 \\
Modelo 2 (sin 11 y 12) & 2330,36 & 134 & 10,741 & $<0,001$ & 0,920 & 0,046 & 0,052
\end{tabular}

Nota. GFI = índice de bondad de ajuste de Joreskog; RMSEA = raíz del error cuadrático medio de aproximación; SRMR = raíz del residuo cuadrático medio estandarizado.

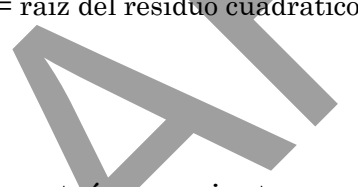

\section{Discusión y Conclusiones}

La EML demostró ser un instrumento de alta consistencia interna, tanto desde la perspectiva del autoconcepto lector como de la valoración de la lectura.

Respecto de los valores obtenidos de consistencia interna, observamos que nuestros resultados son levemente inferiores a los obtenidos por Gambrell et al. (1996) en la validación del instrumento (0,71 y 0,77 versus 0,75 y 0,82 en autoconcepto lector y valor de la lectura, respectivamente). Considerando que alfa de Cronbach depende de la varianza muestral, en el presente estudio la consistencia interna pudo haberse afectado, disminuyendo un poco, por la nula representación de establecimientos municipales, siendo la muestra menos heterogénea en cuanto al tipo de dependencia.

Como mencionamos anteriormente, el ítem 11 ("Me preocupa lo que los otros niños piensen de mi lectura") produce un aporte negativo a la consistencia interna de la escala de autoconcepto. Por ejemplo, la consistencia mejora hasta 0,74 en este constructo si se elimina el ítem. Los estudios previos de validación de la EML (Gambrell et al., 1996; Watkins \& Browning, 2015) no realizaron un análisis del aporte individual de cada ítem a la consistencia global o por subescala, por lo tanto, no es posible contrastar con estos. Una posible explicación de la complejidad que este ítem representa para la consistencia interna del instrumento se puede 
encontrar en la interpretación que los propios alumnos hacen del enunciado. En instancias en las que hemos formulado verbalmente a los alumnos esta pregunta, hemos observado que los niños asocian su autoconcepto lector con la calidad lectora, entendida esta por la medición de dominio lector (Marchant, Recart, Cuadrado \& Sanhueza, 2004) que se realiza en muchos colegios chilenos y que se basa esencialmente en una medida de palabras leídas por minuto y la calidad de la lectura oral (silábica, palabra a palabra, unidades cortas, etc.). Dado que este tipo de evaluación es frecuente en los colegios, es posible pensar que si la visión que los estudiantes tienen respecto de su propia lectura se asocia más a aspectos que son valorados por los otros (como es el caso de la medición de dominio lector que realiza el docente), el ítem refleja más el constructo de valoración de la lectura por parte de terceros que la propia percepción de lector como alguien que disfruta intrínsecamente del acto de leer.

En cuanto a la validez, los análisis realizados confirman la validez de constructo del instrumento, por cuanto los ítems desarrollados responden a lo esperado, a la luz de la teoría en la que se este se sustenta. El ítem 11, que teóricamente forma parte del constructo de autoconcepto lector, en nuestro análisis se asoció al constructo de valoración de la lectura (0,35), mientras que en Gambrell et al. (1996) no se reportaron ítems con baja carga factorial o que cargaran en otro factor. De forma consistente, en el AFC realizado, el ítem 11 no presentó una carga factorial significativa en su constructo teórico, a diferencia de lo observado en Watkins y Browning (2015), donde el ítem carga en autoconcepto lector $(0,75)$. El comportamiento factorial del ítem 11 es consistente con el juicio de expertos, en el que se observó que, de los nueve jueces encuestados, un 53\% lo adscribió a autoconcepto lector y el 47\% restante a valoración de la lectura.

En tanto, el 12 (“Saber leer bien es...”), que teóricamente pertenece al constructo de valor de la lectura, en el AFE cargó en el constructo de autoconcepto lector (0,36); sin embargo, en el AFC esto no sucede, mostrando una carga de 0,34, ligeramente inferior a lo observado en Watkins y Browning $(2015)(0,46)$. En el juicio de expertos, un $87,5 \%$ de ellos lo adscribió a valoración de la lectura, mientras que el $12,5 \%$ lo consideró vinculado a autoconcepto lector.

La revisión de distintos modelos en el AFC mostró que el modelo 2 que mejor representa los constructos teóricos subyacentes es el que excluye los ítems 11 y 12, por cuanto estos no aportan de manera significativa a sus respectivos constructos. Por lo tanto, una sugerencia a considerar, en el caso de aplicar la escala en el contexto chileno o de países hispanoparlantes, sería considerar esta doble adscripción de los ítems en el análisis

En síntesis, la validación de la EML en el contexto chileno contribuye con un instrumento válido e internamente consistente que permite explorar dos componentes motivacionales de la lectura: autoconcepto lector y valor de la lectura. El uso de la EML puede ser con fines de investigación, por ejemplo, su aplicación a gran escala permitiría profundizar en las posibles relaciones entre desempeño lector y factores motivacionales de la lectura. La EML puede ser utilizada también a nivel de aula, poniendo a disposición de los docentes una herramienta de fácil aplicación e interpretación para medir y monitorear estos componentes de motivación. La información recogida permitirá al profesor retroalimentar sus prácticas pedagógicas y tomar decisiones que apunten hacia una mayor motivación por la lectura desde la educación básica. Desde una perspectiva teórica, escalas como la EML permiten evaluar dimensiones relacionadas con la motivación lectora que, al ser desarrolladas en forma más sistemática por los docentes, podrían fortalecer el hábito lector a lo largo de la vida. La relación bidireccional entre motivación lectora y desempeño lector puede favorecer que los lectores chilenos mejoren de manera considerable sus habilidades lectoras (Morgan \& Fuchs, 2007).

Si bien existen otros instrumentos para medir la motivación lectora (e.g., McKenna, Conradi, Lawrence, Jang \& Meyer, 2012; McKenna et al., 1995), muchos se han orientado a la medición de la motivación en el segundo ciclo básico y/o comparando escalas de motivaciones por distintos tipos de lectura (McKenna et al, 2012; Muñoz Valenzuela et al., 2012), por lo que nos parece que la validación de la EML en el contexto nacional contribuye a conocer mejor el comportamiento lector en su dimensión afectiva y de manera más temprana.

Para el contexto chileno en particular, el estudio de las variables motivacionales asociadas a la lectura reviste especial importancia, considerando que numerosos estudios dan cuenta de un desinterés generalizado hacia la lectura. Esta falta de motivación se da a lo largo de prácticamente toda la vida escolar e incluso en la vida adulta - además se observan diferencias de género en desmedro de los hombres-, la que, sin duda, impacta el desempeño en comprensión lectora. 


\section{Limitaciones y Proyecciones}

Una limitación del estudio fue no contar con estudiantes pertenecientes a establecimientos municipales, debido a que no se obtuvieron suficientes consentimientos informados. La muestra habría sido más heterogénea si es que este tipo de alumnos hubiera participado. Sería deseable replicar el estudio en una muestra que incluya estudiantes de estos colegios, resguardando, así, la representatividad de todas las dependencias administrativas de los establecimientos.

Asimismo, creemos que es necesario seguir analizando la escala, por ejemplo, con los ítems modificados por Malloy et al. (2013), trabajo en el cual se realizó una actualización de las afirmaciones de la escala en función de los cambios culturales y lingüísticos desde la creación de la misma. Especialmente, se podría realizar esta revisión considerando ajustes a los ítems 11 y 12, los que mostraron mayor ambigüedad en cuanto a su carga factorial, a fin de que cada uno de ellos se encuadre mejor en el constructo con el cual debiera estar alineado. De la misma manera, sería interesante complementar la versión en español de la EML con la entrevista personal que Gambrell et al. (1996) incluyeron en el perfil original de motivación lectora, la que justamente permite obtener información de tipo cualitativo acerca de las preferencias literarias de los alumnos en distintos géneros de lectura. Esta entrevista contiene preguntas abiertas, pero guiadas con un libreto para que el profesor oriente la conversación. Tal como sostienen las autoras, el objetivo de la entrevista es "generar información que entregue visiones reales de las experiencias lectoras de los alumnos" (Gambrell et al., 1996, p. 525). Este tipo de evidencia puede ser de gran utilidad tanto para investigadores como para docentes, con el fin de comprender mejor la dimensión motivacional de la lectura.

Nos parece importante recomendar, también, que en futuras mediciones de motivación lectora se tome en consideración la lectura digital, pensando que hay una tendencia cada vez mayor a leer en formatos como páginas web, blogs, ebooks y redes sociales. El impacto de estas nuevas formas de alfabetización ya ha sido evaluado en pruebas como PISA a nivel mundial. En nuestro país, sin embargo, la medición de los intereses y hábitos lectores de escolares en medios digitales no ha sido explorada en el contexto escolar, como se desprende, por ejemplo, del estudio realizado por la Agencia de la Calidad de la Educación (2016b), en el que se sostiene que habría algún nivel de asociación entre la motivación lectora y la frecuencia de lectura de textos digitales.

\section{Referencias}

Afflerbach, P. P. \& Cho, B. -Y. (2011). The classroom assessment of reading. En M. L. Kamil, P. D. Pearson, E. B. Moje \& P. P. Afflerbach (Eds.), Handbook of reading research (Vol. IV, pp. 487-514). New York, NY: Routledge.

Agencia de la Calidad de la Educación (2016a). Resultados educativos 2015. Santiago, Chile: Autor.

Agencia de la Calidad de la Educación (2016b). Estudio: factores que influyen en la motivación por la lectura y su relación con logros de aprendizajes e indicadores de desarrollo personal y social. Santiago, Chile: Autor.

Arnold, T. \& García Céspedes, N. (2012). Estudio analiza implementación de Plan Nacional de Fomento de la Lectura. Revista de Educación del Ministerio de Educación de Chile, 351, 27-32.

Becker, M., McElvany, N. \& Kortenbruck, M. (2010). Intrinsic and extrinsic reading motivation as predictors of reading literacy: A longitudinal study. Journal of Educational Psychology, 102, 773-785. https://doi.org/10.1037/a0020084

Bentler, P. M. (1990). Comparative fit indexes in structural models. Psychological Bulletin, 107, 238-246. https://doi.org/10.1037/00332909.107.2.238

Brislin, R. W. (1970). Back-translation for cross-cultural research. Journal of Cross-Cultural Psychology, 1, $185-216$. https://doi.org/10.1177/135910457000100301

Cartwright, K. B., Marshall, T. R. \& Wray, E. (2016). A longitudinal study of the role of reading motivation in primary students' reading comprehension. Implications for a less simple view of reading. Reading Psychology, 37, 55-91. https://doi.org/10.1080/02702711.2014.991481

Chapman, J. W. \& Tunmer, W. E. (1995). Development of young children's reading self-concepts: An examination of emerging subcomponents and their relationship with reading achievement. Journal of Educational Psychology, 87, 154-167. https://doi.org/10.1037//0022-0663.87.1.154

Chile, Ministerio de Educación (2013). El Centro de Recursos para el Aprendizaje: avances y desafíos (Serie Evidencias Año 2 № 23 ). Santiago, Chile: Autor, Centro de Estudios. Extraído de https://centroestudios.mineduc.cl/wp-content/uploads/sites/100/2017/06/A2_N23_CRA.pdf

Collins, J. W. \& O'Brien, N. P. (Eds.) (2003). The Greenwood dictionary of education. Westport, CT: Greenwood Press.

Consejo Nacional de la Cultura y las Artes (2014). Encuesta de comportamiento lector ECL 2014. Santiago, Chile: Autores. Extraído de http://plandelectura.gob.cl/recursos/encuesta-de-comportamiento-lector-2014/

Consejo Nacional de la Cultura y las Artes \& Centro de Microdatos de la Universidad de Chile (2011). Estudio sobre el comportamiento lector a nivel nacional: informe final. Santiago, Chile: Autores. Extraído de http://plandelectura.gob.cl/recursos/estudio-sobrecomportamiento-lector-a-nivel-nacional-2011/

Cumsille, P., Martínez, M. L., Rodríguez, V. \& Darling, N. (2014). Análisis psicométrico de la Escala Parental Breve (EPB): invarianza demográfica y longitudinal en adolescentes chilenos. Psykhe, 23(2), 1-14. https://doi.org/10.7764/psykhe.23.2.665

Deci, E. L. (1992). The relation of interest to the motivation of behavior: A self-determination theory perspective. En K. A. Renninger, S. Hidi \& A. Krapp (Eds.), The role of interest in learning and development (pp. 43-70). Hillsdale, NJ: Lawrence Erlbaum. 
Eccles, J. S. (1983). Expectancies, values, and academic behaviors. En J. T. Spence (Ed.), Achievement and achievement motives: Psychological and sociological approaches (pp. 75-146). San Francisco, CA: Freeman.

Eccles, J. S. \& Wigfield, A. (2002). Motivational beliefs, values and goals. Annual Review of Psychology, 53, 109-132. https://doi.org/10.1146/annurev.psych.53.100901.135153

Fundación La Fuente/Adimark GFK (2010). Chile y los libros 2010. Santiago, Chile: Autor. Extraído de http://www.fundacionlafuente.cl/wpcontent/uploads/2010/11/Chile-y-los-libros-2010_FINAL-liviano.pdf

Gambrell, L. B., Korkeamäki, R. -L., Korkeamäki, R., Tafa, E., Orellana, P., Melo, C. ... Massey, C. (2013, Agosto). A cross-cultural exploration of early reading motivation. Ponencia presentada en la $18^{\text {th }}$ European Conference on Reading, Jönköping, Suecia.

Gambrell, L. B., Palmer, B. M., Codling, R. M. \& Mazzoni, S. A. (1996). Assessing motivation to read. The Reading Teacher, 49, 518-533. https://doi.org/10.1598/RT.49.7.2

Gottfried, A. E. (1985). Academic intrinsic motivation in elementary and junior high school students. Journal of Educational Psychology, 77, 631-645. https://doi.org/10.1037/0022-0663.77.6.631

Guthrie, J. T. \& Klauda, S. L. (2014). Effects of classroom practices on reading comprehension, engagement, and motivations for adolescents. Reading Research Quarterly, 49, 387-416. https://doi.org/10.1002/rrq.81

Guthrie, J. T., Wigfield, A., Barbosa, P., Perencevich, K. C., Taboada, A., Davis, M. H. ... Tonks, S. (2004). Increasing reading comprehension and engagement through concept-oriented reading instruction. Journal of Educational Psychology, 96, 403-423. https://doi.org/10.1037/0022-0663.96.3.403

Guthrie, J. T., Wigfield, A., Metsala, J. L. \& Cox, K. E. (1999). Motivational and cognitive predictors of text comprehension and reading amount. Scientific Studies of Reading, 3, 231-256. https://doi.org/10.1207/s1532799xssr0303_3

Hidi, S. \& Harackiewicz, J. M. (2000). Motivating the academically unmotivated: A critical issue for the $21^{\text {st }}$ century. Review of Educational Research, 70, 151-179. https://doi.org/10.3102/00346543070002151

Hiebert, E. H. (Ed.) (2009). Reading more, reading better. New York, NY: Guilford Press.

Kazelskis, R., Thames, D., Reeves, C., Flynn, R., Taylor, L., Beard, L. A. \& Turnbo, D. (2005). Reliability and stability of Elementary Reading Attitude Survey (ERAS) scores across gender, race, and grade level. The Professional Educator, 27(1-2), 29-37. Extraído de http://wp.auburn.edu/educate/wp-content/uploads/2015/11/combined-fall_04-spring_05.pdf

Kirsch, I., de Jong, J., Lafontaine, D., McQueen, J., Mendelovits, J. \& Monseur, C. (2002). Reading for change: Performance and engagement across countries. Results from PISA 2000. Paris, Francia: Organisation for Economic Co-operation and Development. Extraído de https://www.oecd.org/edu/school/programmeforinternationalstudentassessmentpisa/33690904.pdf

Malloy, J. A. \& Gambrell, L. B. (2008). New insights on motivation in the literacy classroom. En C. C. Block \& S. R. Paris (Eds.), Comprehension instruction: Research-based best practices (2a ed., pp. 226-238). New York, NY: Guilford Press.

Malloy, J. A., Marinak, B. A., Gambrell, L. B. \& Mazzoni, S. A. (2013). Assessing motivation to read: The Motivation to Read ProfileRevised. The Reading Teacher, 67, 273-282. https://doi.org/10.1002/trtr1215

Marchant, T., Recart, I., Cuadrado, B. \& Sanhueza, J. (2004). Pruebas de dominio lector FUNDAR para alumnos de enseñanza básica. Santiago, Chile: Ediciones Universidad Católica de Chile.

McKenna, M. C., Conradi, K., Lawrence, C., Jang, B. G. \& Meyer, J. P. (2012). Reading attitudes of middle school students: Results of a U.S. survey. Reading Research Quarterly, 47, 283-306. https://doi.org/ 10.1002/rrq.021

McKenna, M. C. \& Kear, D. J. (1990). Measuring attitude toward reading: A new tool for teachers. The Reading Teacher, 43, 626-639. https://doi.org/10.1598/RT.43.8.3

McKenna, M. C., Kear, D. J. \& Ellsworth, R. A. (1995). Children's attitudes toward reading: A national survey. Reading Research Quarterly, 30, 934-956. https://doi.org/10.2307/748205

Möller, J., Pohlmann, B., Köller, O. \& Marsh, H. W. (2009). A meta-analytic path analysis of the internal/external frame of reference model of academic achievement and academic self-concept. Review of Educational Research, 79, 1129-1167. https://doi.org/10.3102/0034654309337522

Morgan, P. L. \& Fuchs, D. (2007). Is there a bidirectional relationship between children's reading skills and reading motivation? Exceptional Children, 73, 165-183. https://doi.org/10.1177/001440290707300203

Morgan, P. L., Fuchs, D., Compton, D. L., Cordray, D. S. \& Fuchs, L. S. (2008). Does early reading failures decrease children's reading motivation? Journal of Learning Disabilities, 41, 387-404. https://doi.org/10.1177/0022219408321112

Muñoz Valenzuela, C., Ferreira Torres, S., Sánchez Quintul, P., Santander Pérez, S., Pérez Rodríguez, M. \& Valenzuela Carreño, J. (2012). Características psicométricas de una escala para caracterizar la motivación por la lectura académica. Revista Electrónica de Investigación Educativa, 14(2), 118-132. Extraído de http://www.scielo.org.mx/pdf/redie/v14n2/v14n2a9.pdf

Neuman, S. B. \& Celano, D. (2001). Access to print in low-income and middle-income communities: An ecological study of four neighborhoods. Reading Research Quarterly, 36, 8-26. https://doi.org/10.1598/RRQ.36.1.1

Organisation for Economic Co-operation and Development (2000). Literacy in the information age: Final report of the International Adult Literacy Survey. Paris, Francia: Autor. Extraído de https://www.oecd.org/edu/skills-beyond-school/41529765.pdf

Organisation for Economic Co-operation and Development (2011). Do students today read for pleasure? PISA in Focus, 8, 1-4. Paris, Francia: Autor. Extraído de https://www.oecd.org/pisa/pisaproducts/pisainfocus/48624701.pdf

Organisation for Economic Co-operation and Development (2016). Skills matter: Further results from the Survey of Adult Skills. Paris, Francia: Autor. https://doi.org/10.1787/9789264258051-en

Pintrich, P. R. \& Schunk, D. H. (2002). Motivation in education: Theory, research, and applications (2a ed.). Upper Saddle River, NJ: Prentice Hall.

Rosseel, Y. (2012). lavaan: An R package for structural equation modeling. Journal of Statistical Software, 48(2), 1-36. https://doi.org/10.18637/jss.v048.i02

Santa, C. M., Williams, C. K., Ogle, D., Farstrup, A. E., Au, K. H., Baker, B. M. ... Shanahan, T. (2000). Excellent reading teachers: A position statement of the International Reading Association. Journal of Adolescent \& Adult Literacy, 44, 193-199. Extraído de http://www.jstor.org/stable/40013543

Schaffner, E., Philipp, M. \& Schiefele, U. (2016). Reciprocal effects between intrinsic reading motivation and reading competence? A cross-lagged panel model for academic track and nonacademic track students. Journal of Research in Reading, 39, 19-36. https://doi.org/10.1111/1467-9817.12027

Schiefele, U., Schaffner, E., Möller, J. \& Wigfield, A. (2012). Dimensions of reading motivation and their relation to reading behavior and competence. Reading Research Quarterly, 47, 427-463. https://doi.org/10.1002/RRQ.030 
Schunk, D. H. (1985). Self-efficacy and classroom learning. Psychology in the Schools, 22, 208-223. https://doi.org/10.1002/15206807(198504)22:2<208::AID-PITS2310220215>3.0.CO;2-7

Schunk, D. H., Pintrich, P. R. \& Meece, J. L. (2008). Motivation in education: Theory, research, and applications (3a ed.). Upper Saddle River, NJ: Pearson/Merrill Prentice Hall.

Taboada, A., Tonks, S. M., Wigfield, A. \& Guthrie, J. T. (2009). Effects of motivational and cognitive variables on reading comprehension. Reading and Writing, 22, 85-106. https://doi.org/10.1007/s11145-008-9133-y

Uguroglu, M. E. \& Walberg, H. J. (1979). Motivation and achievement: A quantitative synthesis. American Educational Research Journal, 16, 375-389. https://doi.org/10.3102/00028312016004375

Unrau, N. \& Schlackman, J. (2006). Motivation and its relationship with reading achievement in an urban middle school. The Journal of Educational Research, 100, 81-101. https://doi.org/10.3200/JOER.100.2.81-101

Van Slyck, A. A. (1995). Free to all: Carnegie libraries \& American culture, 1890-1920. Chicago, IL: University of Chicago Press.

Wang, J. H. -Y. \& Guthrie, J. T. (2004). Modeling the effects of intrinsic motivation, extrinsic motivation, amount of reading, and past reading achievement on text comprehension between U.S. and Chinese students. Reading Research Quarterly, 39, $162-186$. https://doi.org/10.1598/RRQ.39.2.2

Watkins, M. W. \& Browning, L. J. (2015). The Baylor revision of the Motivation to Read Survey (B-MRS). Research and Practice in the Schools, 3(1), 37-50. Extraído de http://edpsychassociates.com/Papers/Bmrs(2015).pdf

Widaman, K. F., Ferrer, E. \& Conger, R. D. (2010). Factorial invariance within longitudinal structural equation models: Measuring the same construct across time. Child Development Perspectives, 4, 10-18. https://doi.org/10.1111/j.1750-8606.2009.00110.x

Wigfield, A. \& Guthrie, J. T. (1997). Relations of children's motivation for reading to the amount and breadth of their reading. Journal of Educational Psychology, 89, 420-432. https://doi.org/10.1037/0022-0663.89.3.420

\section{Anexo}

\section{Perfil de Motivación para Leer: Escala de Lectura}

Colegio

Fecha

Curso

1. Mis amigos creen que yo soy

$\mathrm{O}$ un muy buen lector

O un buen lector

$\mathrm{O}$ un lector medio

O un mal lector

2. Leer un libro es algo que a mí me gusta hacer

$\mathrm{O}$ nunca

O no muy seguido

$\mathrm{O}$ a veces

$\mathrm{O}$ muy seguido

3. Yo leo

$\mathrm{O}$ no tan bien como mis amigos

$\mathrm{O}$ casi igual que mis amigos

$\mathrm{O}$ un poquito mejor que mis amigos

$\mathrm{O}$ bastante mejor que mis amigos

4. Mis mejores amigos piensan que leer es

O muy entretenido

O entretenido

O aceptable

O aburrido

5. Cuando me encuentro con una palabra que no conozco, puedo

O casi siempre lograr entenderla

$\mathrm{O}$ a veces la logro entender

O casi nunca la logro entender

O nunca la logro entender 
6. Yo le cuento a mis amigos sobre los buenos libros para leer.

$\mathrm{O}$ nunca lo hago

$\mathrm{O}$ casi nunca lo hago

O lo hago algunas veces

O lo hago mucho

7. Cuando leo solo, comprendo

O casi todo lo que leo

$\mathrm{O}$ algo de lo que leo

O casi nada de lo que leo

O nada de lo que leo

8. Las personas que leen mucho son

O muy interesantes

$\mathrm{O}$ interesantes

$\mathrm{O}$ poco interesantes

O aburridas

9. Yo soy

O un mal lector

O un lector aceptable

$\mathrm{O}$ un buen lector

$\mathrm{O}$ un muy buen lector

10. Yo creo que las bibliotecas son

O un gran lugar para pasar el tiempo

$\mathrm{O}$ un lugar interesante para pasar el tiempo

O un lugar aceptable para pasar el tiempo

$\mathrm{O}$ un lugar aburrido para pasar el tiempo

11. Me preocupa lo que los otros niños piensen de mi lectura

O todos los días

O casi todos los días

O de vez en cuando

$\mathrm{O}$ nunca

12. Saber leer bien es

O no muy importante

O más o menos importante

O importante

O muy importante

13. Cuando el profesor me pregunta sobre lo que yo he leído

$\mathrm{O}$ no se me ocurre qué responder

O me cuesta pensar lo que voy a responder

$\mathrm{O}$ a veces pienso en una respuesta

O siempre pienso en una respuesta

14. Creo que leer es

O una forma aburrida de pasar el tiempo

O una forma aceptable de pasar el tiempo

O una forma interesante de pasar el tiempo

O una forma grandiosa de pasar el tiempo 


\section{Leer es}

O muy fácil para mí

O más o menos fácil para mí

O más o menos difícil para mí

O muy difícil para mí

16. Cuando sea grande yo

O no voy a pasar tiempo leyendo

O voy a pasar poco tiempo leyendo

O voy a pasar algún tiempo leyendo

O voy a pasar mucho tiempo leyendo

17. Cuando estoy en un grupo contando historias

O casi nunca hablo de mis ideas

O algunas veces hablo de mis ideas

O casi siempre hablo de mis ideas

O siempre hablo de mis ideas

18. Me gustaría que mi profesor nos leyera libros en voz alta en clase

O todos los días

O casi todos los días

$\mathrm{O}$ de vez en cuando

O nunca

19. Cuando yo leo en voz alta, leo

O muy mal

O más o menos

$\mathrm{O}$ bien

O muy bien

20. Cuando me regalan un libro, me siento

O muy feliz

O contento

$\mathrm{O}$ no me siento muy contento

O no me siento feliz

\section{Hoja de Puntaje Escala de Lectura}

Nombre estudiante:

Grado:

Profesor:

Fecha administración:

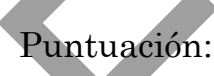

$1=4$

$2=3$

$3=2$

$4=1$ 
Autoconcepto lector

*recodificar

recodificar

*recodificar

*recodificar

*recodificar

\section{1.}

3.

5.

7.

9.

11.

13.

15.

17.

19.
Valor de la lectura

$\begin{array}{lrl} & 2 . & \\ \text { *recodificar } & 4 . & - \\ & 6 . & - \\ \text { *recodificar } & 8 . & - \\ \text { *recodificar } & 10 . & - \\ & 12 . & - \\ & 14 . & - \\ & 16 . & - \\ \text { *recodificar } & 18 . & -\end{array}$

Autoconcepto puntuación original Valor puntuación original

Puntuación original total de la escala (autoconcepto y valor)

Porcentaje puntajes

Autoconcepto

Valor

Comentarios

Escala total

Fecha de recepción: Agosto de 2016.

Fecha de aceptación: Julio de 2017. 